\title{
Découverte d'un nouveau gisement du Magdalénien moyen à Enval (Vic-le-Comte, Puy-de-Dôme)
}

\author{
Frédéric Surmely, Sandrine Costamagno, Jay Franklin, Maureen Hays, \\ avec la collaboration de François Chevrier, Stéphane Madeleine, \\ Gérard Vernet et Ilaria PATANia
}

\section{Circonstances de la découverte}

À moins de $2 \mathrm{~km}$ du val d'Allier, le village d'Enval est installé sur un escarpement gréseux haut de plus de $40 \mathrm{~m}$ et long de plus de $1 \mathrm{~km}$, qui comprend plusieurs lignes étagées de petits abris-sous-roche dans une topographie, hélas, fortement transformée par l'urbanisation. La première mention de découverte de vestiges préhistoriques date de 1877. Une des dernières découvertes fit l'objet d'une fouille programmée (abri Durif : Bourdelle et Merlet, 1991) et, en 1997, un habitant, à l'occasion de travaux, découvrit un nouveau locus malheureusement limité à un lambeau de couche (Enval 2 : Surmely et al., 1997).

En 2009 débuta un programme de recherche destiné à étudier l'ensemble du mobilier des fouilles Bourdelle et à évaluer le potentiel du lieu. L'ensemble des occupations a ainsi pu être rattaché au Magdalénien moyen (Surmely, 2011; Merlet et al., sous presse).

Le nouveau locus décrit ici se situe à l'extrémité nord du village, à environ $100 \mathrm{~m}$ des lieux de découvertes antérieures. C'est lors de travaux de terrassement à la base de l'escarpement rocheux que, en mars 2016, le propriétaire du terrain, M. Moliard, mit au jour des vestiges préhistoriques. Il alerta le service régional de l'Archéologie et une opération de nettoyage et d'expertise fut rapidement mise en place grâce à son accord.

Cette tâche fut réalisée sur une dizaine de jours avec une équipe d'étudiants de l'East Tennessee University (USA) et le soutien de la municipalité, du propriétaire, de riverains et de l'association « Terre ancienne ». Nous avons effectué la mise en sécurité provisoire du site, le tamisage des déblais du terrassement, deux sondages manuels en avant de l'escarpement, le redressement et le relevé de la coupe frontale, le relevé topographique et le prélèvement d'échantillons. Nous donnons ici un premier aperçu des découvertes, alors que les études sont en cours.

\section{Organisation générale et extension du site}

Le site est un abri-sous-roche s'ouvrant à la base d'un banc gréseux. De gros blocs présents dans le remplissage témoignent de l'effondrement du toit et donc de son recul depuis le Magdalénien. Le surplomb a aussi très probablement été en partie retaillé à une époque historique; s'y ajoutent des perturbations à l'avant. La superficie couverte par le surplomb rocheux au Magdalénien reste donc difficile à évaluer. La surface restante du site est estimée à environ $15 \mathrm{~m}^{2}$.

Au niveau de la coupe frontale principale, la puissance des niveaux archéologiques avoisine $140 \mathrm{~cm}$, avec au moins quatre niveaux sédimentaires distincts (unités stratigraphiques : US 40, 70, 80 et 100) livrant de très nombreux vestiges magdaléniens (fig. 1). Les nombreuses connexions osseuses suggèrent l'absence de remaniement post-dépositionnel important, mais ces premières observations restent à confirmer. Les retombées volcaniques sont absentes.

\section{Industrie lithique}

\section{Matières premières}

Les matières allochtones représentent toujours plus de la moitié du poids total de l'industrie lithique taillée, proportion qui atteint $73 \%$ dans l'US40 et qui apparaît très élevée en comparaison de la séquence du Sol de la Grange de l'abri Durif (27\% du matériel lithique : Surmely, 2010). La diversité des types de silex apparaît remarquable (cette analyse s'appuie sur les travaux et la lithotèque issus du programme de recherche sur les matériaux siliceux de la région Centre : Surmely, 2008). Outre les habituels silex «blonds » de la craie du Turonien inférieur, composante principale de l'approvisionnement, et les silex du Turonien supérieur et moyen du secteur du Grand-Pressigny, on note la présence, inédite en Auvergne, de silex crétacés bariolés du secteur de Fontmaure (Véllèches, Vienne : Pradel, 1967) à une distance à vol d'oiseau de $260 \mathrm{~km}$ ! De petits éclats de cristal de roche (quartz hyalin) sont aussi présents; leur origine reste inconnue, mais pourrait se situer dans le domaine alpin.

\section{Technotypologie}

L'objectif général est une production laminaire de qualité, avec des préparations soignées comme les talons en éperon. La mise en œuvre de chaînes opératoires différenciées est un trait habituel dans le val d'Allier (Surmely, 2002 et 2008).

Les silex locaux ont été introduits à l'état de blocs bruts et de préformes grossièrement aménagées, exploités sur place. Le taux de transformation des supports en outils est assez faible (4 à $12 \%$ selon les niveaux).

La chaîne opératoire est très différente pour les silex allochtones, introduits à l'état de préformes. La gestion de ces matériaux est orientée vers la production de supports allongés, mais avec cette fois la recherche d'une rentabilité maximale, se traduisant par un très fort taux de transformation des supports et des ravivages d'outils jusqu'à exhaustion. La longueur de nombreux outils avoisine les $2 \mathrm{~cm}$, à la limite de la maniabilité! Les nucléus sont rares et de petite taille. C'est assurément de ce souci 
d'économie que découlent certaines caractéristiques « typologiques », notamment l'assez fort taux d'outils mixtes et multiples et la morphologie des burins. Ceuxci sont en effet dominés par des formes dièdres à biseau assez large, résultant du ravivage multiple de burins ou même d'autres outils.

La production lamellaire a été effectuée sur des nucléus véritables : on n'observe pas de procédés de détachement sur supports ou fragments de supports.

L'outillage est largement dominé par les burins. Armatures microlithiques (lamelles à dos simple), grattoirs et pièces esquillées sont présents dans des proportions moindres et qui varient selon les niveaux. La faible proportion d'armatures microlithiques est remarquable car elle se démarque des chiffres de l'abri Durif (Surmely, 2010) et des autres sites magdaléniens du val d'Allier (Surmely et al., 2002). Les quatre niveaux individualisés présentent des caractéristiques assez proches, qui plaident en faveur d'une étroite proximité fonctionnelle et chronologique.

\section{Archéozoologie}

La faune est extrêmement abondante (environ 10000 pièces) et bien préservée, ce qui est plutôt rare dans la région. L'analyse a été menée sur tous les os déterminables d'un point de vue taxinomique, soit environ 2800 restes.
Les taxons identifiés témoignent du caractère strictement pléistocène des assemblages.

Le spectre faunique est assez homogène sur toute la séquence avec quatre taxons prédominants : renne, bovinés, cheval et lagomorphe. Majoritaire dans toutes les US, le renne représente entre 49,5 et $89,4 \%$ des restes déterminés. Viennent ensuite les lagomorphes avec 9 à $27,4 \%$. L'US 100 livre la plus forte proportion de lagomorphes et se distingue des US sus-jacentes par une plus grande fréquence de gros ongulés, bison et cheval, présents chacun aux environs de $10 \%$. Ces spectres fauniques sont assez comparables à celui d'Enval 2 (Surmely et al., 1997).

Plusieurs ossements montrent des traces anthropiques et les os longs des ongulés présentent systématiquement des cassures sur os frais. Ces éléments militent en faveur d'une origine anthropique des ongulés. L'impact des carnivores semble limité. Seuls les restes de lagomorphes pourraient avoir une origine majoritairement naturelle.

Fait remarquable, les profils squelettiques montrent que tous les éléments sont présents, y compris les vertèbres, ce qui pourrait témoigner de l'introduction de carcasses entières, en particulier pour le renne. Un fragment de crâne d'un renne mâle dépourvu de bois dans l'US 100 indique que l'animal a été abattu à la mauvaise saison. La présence de quelques restes de fœtus évoque également l'hiver.

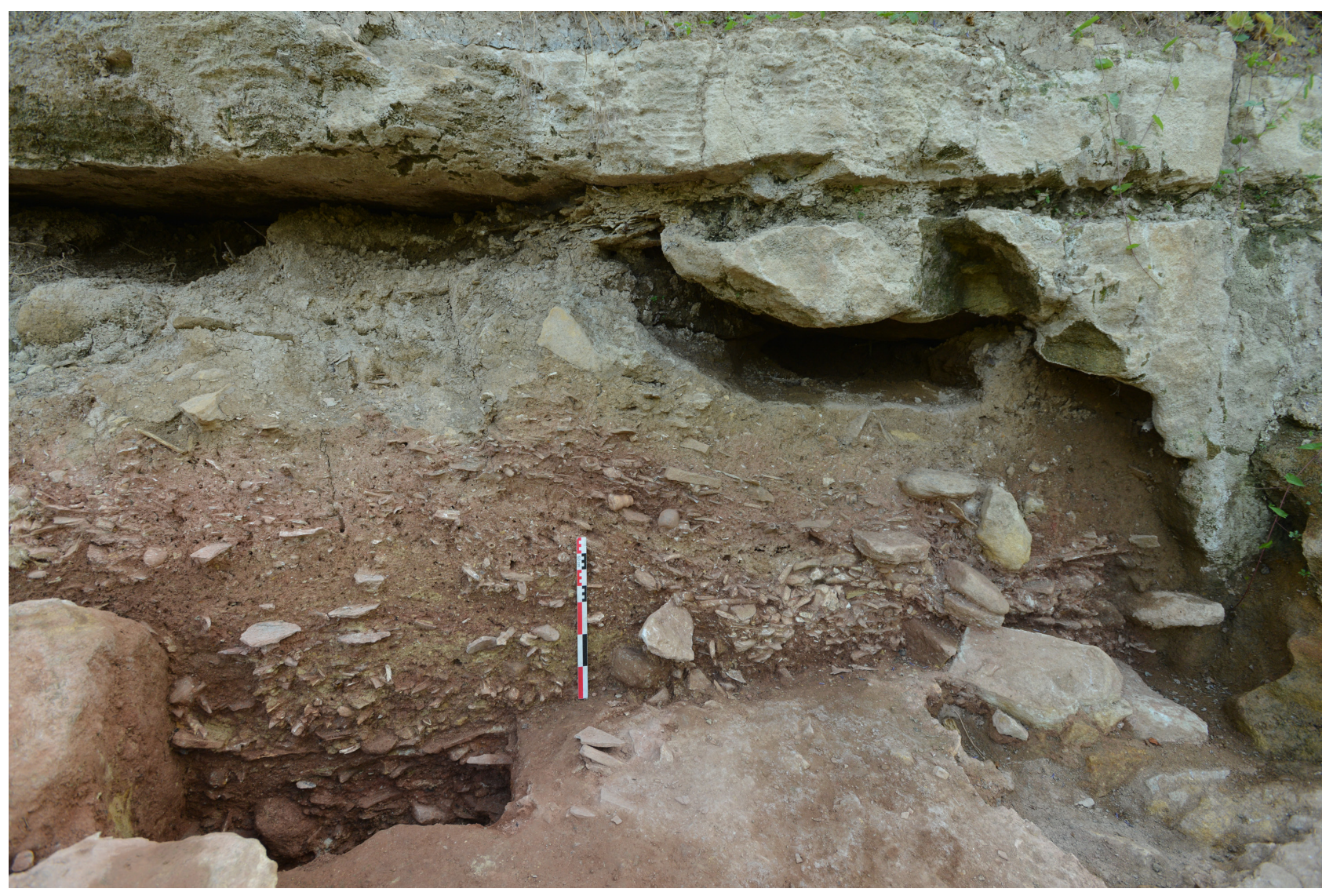

Fig. 1 - Vue partielle de la coupe frontale, après nettoyage et redressement, montrant les quatre niveaux d'occupation et l'extrême abondance des restes lithiques et surtout osseux (cliché F. Surmely). 


\section{Objets de parure}

Quarante-sept objets de parure ont été recueillis et se partagent en plusieurs catégories (fig. 2). Les coquillages ( $n=14)$ sont tous des fossiles marins, issus très probablement des faluns de Touraine. Les espèces représentées ne sont pas rares et même pour certaines communes. Certaines pièces portent des traces d'usure importante. En comparaison, l'abri Durif n'avait livré qu'une seule pièce, une ammonite percée (Surmely, 2013; Merlet et al., sous presse).

Le site a aussi livré de nombreuses dents percées $(\mathrm{n}=17)$, objets très rares à l'abri Durif. Elles proviennent d'animaux variés (cheval, renne, cerf, bouquetin, marmotte, boviné), avec une prédominance du renne $(n=6)$ et du cheval $(n=5)$. La présence de la marmotte est singulière et évoque les sites de l'arc jurassien (Poplin, 1983a; Büllinger, 2005). Les nombreuses incisives de renne isolées et non perforées retrouvées sur le site pourraient être des réserves de pièces brutes, avec un choix porté sur des dents d'individus âgés, voire très âgés. Sur presque toutes les dents percées $(n=12)$, la partie proximale de la racine a été sciée. La troncature est en général très poussée, au point que la perforation a été placée immédiatement sous le collet. La présence d'une chute (apex de racine) témoigne d'une fabrication sur place, au moins partielle. Trois perles circulaires en lignite ont également été découvertes, ainsi qu'un déchet de façonnage.
Enfin, le site a livré de petits rognons de silex et pisolithes dont la provenance, allochtone (formations sédimentaires du Crétacé supérieur), pourrait être la même que celle des matériaux utilisés pour l'industrie lithique. Ces pièces ne présentent aucun stigmate d'utilisation visible. La forme de l'un d'eux évoque grossièrement une silhouette féminine.

\section{Industrie osseuse}

Les différentes étapes de la chaîne opératoire de l'industrie osseuse sont représentées. Parmi les fragments de bois de renne découverts, certains sont des déchets d'extraction de baguettes et d'autres des produits, principalement des baguettes. Quelques ossements portent des traces de rainurage et un fragment de diaphyse de grand ongulé a été utilisé comme retouchoir. Les objets finis sont rares : une petite sagaie à cannelure centrale et un apex de grosse aiguille ou petite sagaie.

\section{Art mobilier}

Deux fragments de plaquettes (arkose locale) portent des gravures représentant des portions de corps animal. Ces témoins d'art mobilier sont à rapprocher de ceux découverts à l'abri Durif (Merlet et al., sous presse).

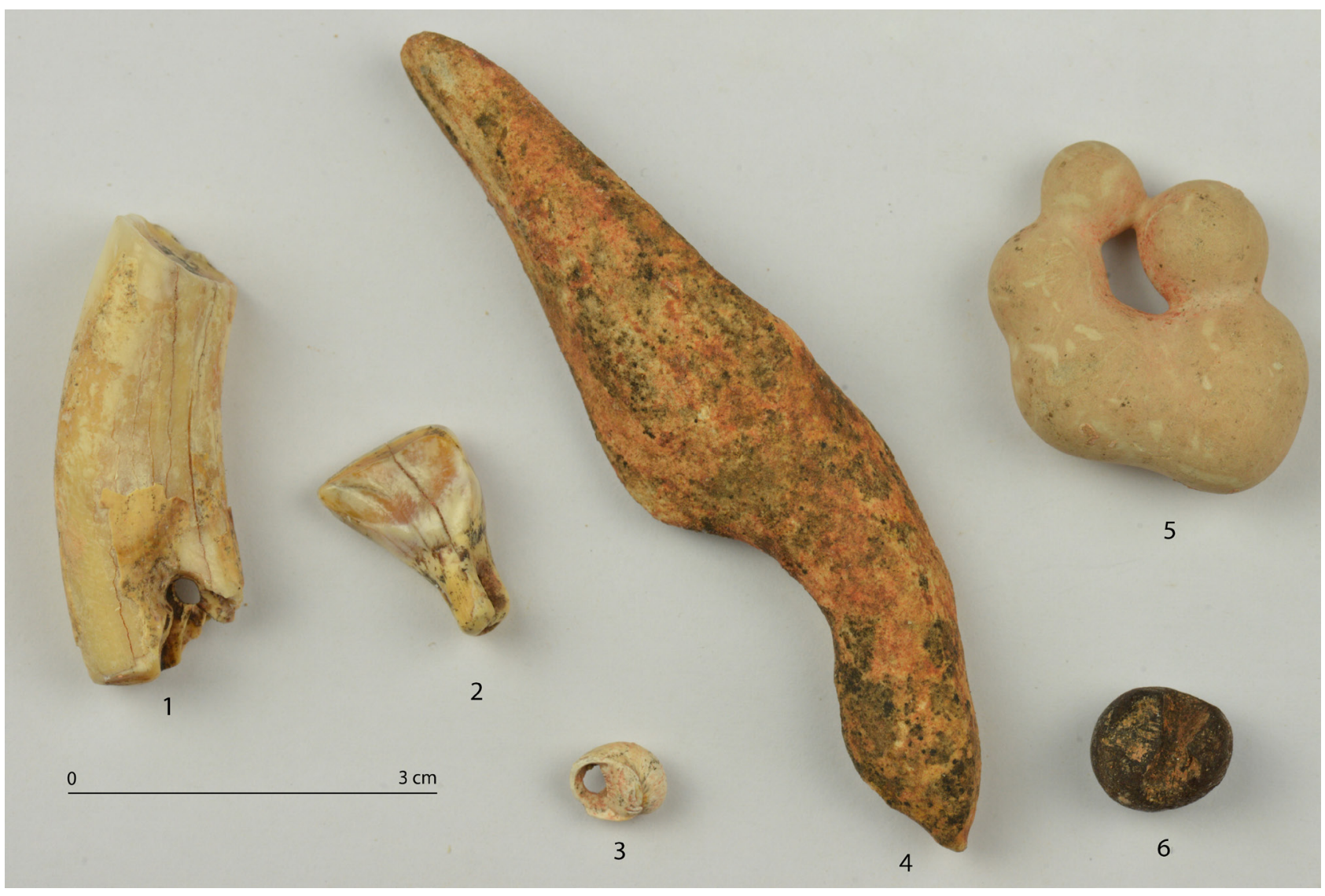

Fig. 2 - Éléments de parure de types divers provenant de l’US $80.1: \mathrm{n}^{\circ} 11$ (incisive de cheval tronquée et perforée); $2: \mathrm{n}^{\circ} 17$ (incisive de cerf sciée et perforée); $3: \mathrm{n}^{\circ} 18$ (coquille de Natica percée); $4: \mathrm{n}^{\circ} 20$ (rognon de calcaire silicifié, possible FFS); $5: \mathrm{n}^{\circ} 20$ (petit nodule de calcaire silicifié ocré avec trou naturel); $6: \mathrm{n}^{\circ} 15:$ fragment de perle en lignite (cliché F. Surmely). 


\section{Datations}

Quatre dates ${ }^{14} \mathrm{C}$ par AMS ont été faites sur des os non brûlés : US 70, $13810 \pm 30$ BP (SacA46130); US 80, 13779 \pm 40 BP (D-AMS 018147); US 100, $13851 \pm 40$ BP (D-AMS 18788); US 40, $13851 \pm 63$ BP (D-AMS 020378). Ces résultats placent l'occupation du site dans une fourchette assez restreinte, à la fin du Magdalénien moyen selon la chronologie de Langlais et al. (2016).

\section{Conclusion}

Ce site se place dans la continuité topographique des découvertes précédentes. On peut supposer qu'il existait une occupation magdalénienne continue, sur plus de $150 \mathrm{~m}$ de longueur, au pied des escarpements dominant le ruisseau. Il est également possible que la station s'étende plus à l'ouest sous les colluvions récentes. La stratigraphie montre l'existence de plusieurs niveaux archéologiques très riches, séparés par des horizons stériles ou presque stériles. Les dates ${ }^{14} \mathrm{C}$ permettent de rattacher ces niveaux à la phase finale du Magdalénien moyen, tout comme les autres occupations reconnues à Enval (Angevin et Surmely, 2013). Les études préliminaires réalisées sur la faune et l'industrie lithique font apparaître la singularité manifeste du site, avec notamment des indices de fréquentation en période hivernale. Le mobilier témoigne de liens avec des régions situées à plusieurs centaines de kilomètres. Le site d'Enval-Moliard est d'intérêt exceptionnel pour la connaissance du peuplement du val d'Allier au Paléolithique supérieur. Les premiers résultats du nettoyage réalisé en 2016 demandent à être affinés et confirmés par une fouille véritable, envisagée dès 2017 , qui permettra de réaliser une vraie analyse spatiale et mobilisera une équipe pluridisciplinaire avec des moyens adaptés.

Remerciements : Les auteurs tiennent à remercier les personnes qui ont apporté un soutien précieux à l'opération : Didier Moliard, Gaëlle Le Guisquet, Axel et Florence Winel, Roland Blanchet, Jean-Baptiste Chalin, Mickaël Tournadre, Robert Linam, Davide Mackres, Caroline Bruyère, Lauren Woelkers, Marc Laroche, Yannick Miras et Hervé Delhoofs. Remerciements également à Jean-Marc Pétillon.

\section{RÉFÉRENCES BIBLIOGRAPHIQUES}

Angevin R., Surmely F. (2013) - Le Magdalénien moyen et la trajectoire historique des sociétés du $\mathrm{XVI}^{\mathrm{e}}$ millénaire av. J.-C. en France centrale, Comptes rendus Palevol, 12, p. 57-68.

Bourdelle Y., Merlet J.-C. (1991) - Le site d'Enval, commune de Vic-le-Comte (Puy-de-Dôme), Bulletin de la société préhistorique française, 88, p. 109-113.

Langlais M., Sécher A., Caux S., Delvigne V., Gourc L., Normand C., SAnchez de la Torre M. (2016) - Lithic Tool Kits: A Metronome of the Evolution of the Magdalenian in Southwest France (19,000-14,000 BP), Quaternary International, 30, p. 1-16.

Merlet J.-C., Plassard F., Chauvière F.-X. (sous presse) L'art mobilier et la parure du gisement magdalénien d'Enval (Vic-le-Comte, Puy-de-Dôme), in L'art au quotidien. Objets ornés du paléolithique supérieur, actes du colloque du musée national de Préhistoire (Les Eyzies, 2014).

Pradel L. (1967) - Les gisements de Fontmaure, Toulouse, Travaux de l'Institut d'art préhistorique, $150 \mathrm{p}$.

Surmely F., Pasty J.-P., Alix P., Murat R., Liabeuf R. (2002) - Le gisement magdalénien du Pont-de-Longues (Les-Martres-de-Veyre, Puy-de-Dôme), Bulletin de la Société préhistorique française, 99, p. 13-38.

Surmely F. (2008) - La diffusion des silex crétacés dans le centre du Massif central durant la Préhistoire (Paléolithique, Mésolithique, Néolithique), Paléo, 20, p. 115-144.

SuRmely F. (2009, 2010, 2011 et 2013) - Rapports sur le programme de recherches sur le site magdalénien d'Enval, service régional de l'Archéologie d'Auvergne, ClermontFerrand.

Surmely F., Fontana L., Liabeuf R., Bourdelle Y. (1997) Nouveaux éléments apportés à l'étude du site d'Enval et du peuplement magdalénien en Limagne, Bulletin de la Société préhistorique française, 94, p. 172-181.

Frédéric Surmely

DRAC Auvergne-Rhône-Alpes, Clermont-Ferrand, UMR 6042 du CNRS surmely.frederic@wanadoo.fr

Sandrine Costamagno université Toulouse Jean-Jaurès UMR 5608 du CNRS TRACES, Toulouse

Jay FrankLIN East Tennessee University, Johnson City, USA

Maureen Hays College of Charleston, Charleston, USA 